\title{
PHYSICS OF RESONANT TUNNELLING
}

\author{
F.W. Sheard and T.M. Fromhold
}

Department of Physics, University of Nottingham, Nottingham NG7 2RD, England

\begin{abstract}
A description is given of resonant tunnelling processes in double-barrier semiconductor heterostructures from the point of view of sequential theory. If a magnetic field is applied in the plane of the barrier interfaces, the energy and transverse momentum of the electrons injected into the quantum well through the emitter tunnel barrier can be varied independently by changing the applied voltage and magnetic field. This technique can be used to probe the energy and momentum spectrum of the subband states in a quantum well. In $n$-type wide-well structures, a detailed interpretation of magneto-oscillations in the tunnel current has been given in terms of semiclassical orbits of electrons in the well. In $p$-type structures with narrow wells, the dispersion curves of hole subbands, which are complicated by the. strong mixing of light hole and heavy hole states, have been directly studied. Resonant tunnelling into the bound state of single donor atoms in a quantum well has recently been observed. The magnetic field dependence of the tunnel current then gives a measure of the transverse momentum distribution and hence lateral extent of the donor wave function.
\end{abstract}

PACS numbers: 73.20.Dx

\section{Introduction}

Tunnelling through a potential barrier is a basic feature of quantum mechanics since it is a manifestation of the wave nature of matter. The description of alpha particle emission by radioactive nuclei is an early success of tunnelling theory. But in nuclear physics it is difficult to directly influence the tunnel process since the energy barriers are very high $(\approx 10 \mathrm{MeV})$ and very narrow $\left(\approx 10^{-14} \mathrm{~m}\right)$. In semiconductor heterostructures the barriers are much lower $(\approx 100 \mathrm{meV})$ and wider $(\approx 10 \mathrm{~nm})$ so that the tunnel process can be studied by means of the effect of external fields. An electric field, applied parallel to the tunnelling direction, can be used to change the kinetic energy of a tunnelling electron, whilst a magnetic field, applied perpendicular to the tunnelling direction, changes the direction or transverse momentum of a tunnelling electron.

Resonant tunnelling is of particular interest [1]. In this case an electron tunnels through two barriers between which there is a quantum well (QW) which supports a quasibound state. When the energy of the incident electron coincides 


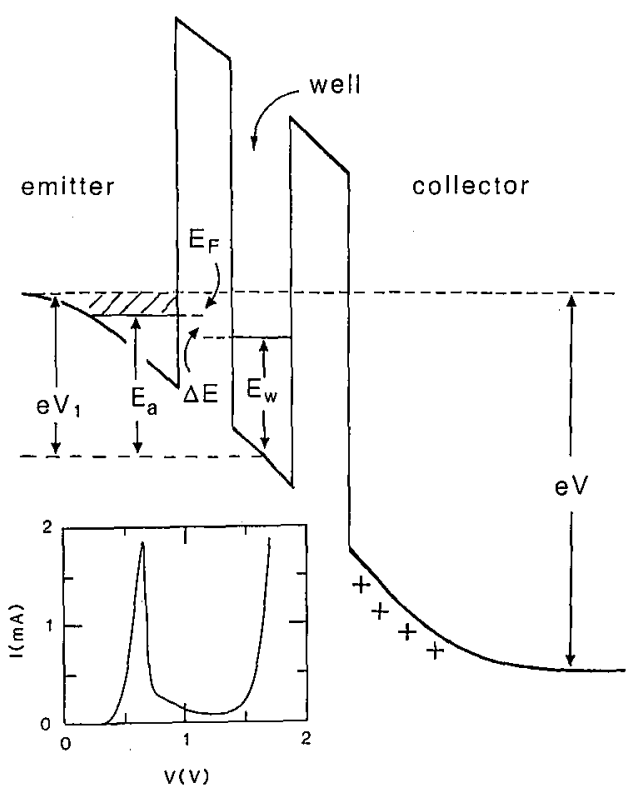

Fig. 1. Spatial variation of electron potential energy through double-barrier heterostructure showing formation of degenerate 2DEG, with Fermi energy $E_{\mathrm{F}}$, in emitter. Lower inset shows current - voltage $I(V)$ characteristic for a symmetric GaAs/(AlGa)As structure with well width $5 \mathrm{~nm}$, barrier width $5.6 \mathrm{~nm}$ and weakly doped emitter and collector contacts, measured at $4 \mathrm{~K}$.

with that of the quasibound state the transmission coefficient has a resonant maximum. Under an external bias voltage the quasibound state is lowered through the range of incident energies giving a resonant peak in the current-voltage $I(V)$ characteristics (Fig. 1). The associated region of negative differential resistance in the $I(V)$ curve gives rise to the possibility of using such resonant tunnelling double-barrier structures (DBS's) in electronic devices [2].

To minimise scattering effects due to dopant diffusion, double-barrier heterostructures are often grown with weakly doped $\left(\approx 10^{16} \mathrm{~cm}^{-3}\right.$ for GaAs $)$ emitter and collector contacts and may also have undoped spacer layers adjacent to the barriers. Under bias an accumulation layer and bound state is formed at the emitter barrier interface and, at liquid helium temperatures, the associated two-dimensional electron gas (2DEG) is degenerate. Tunnelling then occurs between 2D emitter states into the 2D states of the subband in the QW, as shown in Fig. 1. For plane interfaces, the transverse components of momenta, and hence transverse kinetic energy, are conserved in tunnelling. Since total energy is also conserved the resonant condition is achieved when the energy $E_{\mathrm{a}}$ of the bound state in the accumulation layer is equal to the energy $E_{\mathrm{w}}$ of the bound state in the quantum well. At low temperatures this gives rise to a sharp resonant peak in $I(V)$ (see inset in Fig. 1), although there are of course finite level broadening 
eflects [3] due to scattering processes or structural inhomogeneities.

For the interpretation of experiments it is instructive to think of resonant tunnelling as a sequential two-stage process [4]. The electrons first tunnel from the emitter into the bound state of the QW and, owing to scattering processes, lose phase coherence before tunnelling out through the collector barrier. The tunnelling in and tunnelling out processes are regarded as statistically independent transitions. As pointed out by Luryi [4], the resonant feature is retained in the sequential approach since the transition through the emitter barrier requires coincidence of the incident electron energy with the energy of the bound state in the QW.

In this review we shall be principally concerned with the effect of a transverse magnetic field (parallel to the barrier interfaces) on the tunnel current. This has proved to be a useful spectroscopic tool for studying the energy and momentum spectrum of states in a QW and also the way in which these states are modified by electric and magnetic fields.

\section{Effect of magnetic field on electronic motion}

The basic principles are most simply elucidated semiclassically [5]. Taking the $x$ axis to be the tunnelling direction (perpendicular to the interfaces) and the transverse magnetic field $\boldsymbol{B}$ along the $z$ direction, the equation of motion of the transverse momentum component $m^{*} v_{y}$ of an electron (effective mass $m^{*}$, charge $-e$ ) is

$$
m^{*} \frac{\mathrm{d} v_{y}}{\mathrm{~d} t}=e v_{x} B=e B \frac{\mathrm{d} x}{\mathrm{~d} t} .
$$

In the Landau gauge, the vector potential is $\boldsymbol{A}=(0, B x, 0)$, so Eq. (1) gives

$$
\frac{\mathrm{d}}{\mathrm{d} t}\left(m^{*} v_{y}-e B_{x}\right)=\frac{\mathrm{d}}{\mathrm{d} t}\left(m^{*} v_{y}-e A_{y}\right)=\frac{\mathrm{d} p_{y}}{\mathrm{~d} t}=0,
$$

showing that the canonical momentum component $p_{y}$ is a constant of the motion (as also is $p_{z}=m^{*} v_{z}$ ). In quantum mechanics the wave function $\psi(x, y, z)$ is an eigenfunction of canonical momentum operators $\hat{p}_{y}, \hat{p}_{z}$ with eigenvalues $\hbar k_{y}, \hbar k_{z}$ and can be written

$$
\psi(x, y, z)=\psi(x) \operatorname{expi}\left(k_{y} y+k_{z} z\right)
$$

where $\psi(x)$ describes motion in the tumnelling direction and the wave vectors $k_{y}$, $k_{z}$ are conserved throughout the structure.

The magnetic field also gives rise to a force in the tunnelling direction

$$
F_{x}=-e v_{y} B=-\omega_{\mathrm{c}}\left(e B x+p_{y}\right)=-m^{*} \omega_{\mathrm{c}}^{2}(x-X)=-\partial U_{\mathrm{m}} / \partial x
$$

which can be written as the gradient of a simple harmonic potential $U_{\mathrm{m}}(x)=$ $1 /\left(2 m^{*}\right) \omega_{\mathrm{c}}^{2}(x-X)^{2}$, where $\omega_{\mathrm{c}}=e B / m^{*}$ and $X=-\hbar k_{y} / e B$ is the orbit centre of the classical trajectory in the magnetic field. The wave function $\psi(x)$ is hence determined by a one dimensional Schrödinger equation which contains the magnetic potential $U_{\mathrm{m}}(x)$ and also the heterostructure potential energy function $E_{\mathrm{c}}(x)$ of an electron at the conduction band edge. In a bulk semiconductor with $E_{\mathrm{c}}(x)=$ const., we have simple harmonic oscillator eigenfunctions $\psi_{n}(x)(n=0,1,2 .$.$) and$ the eigenvalues are the well-known Landau levels $E_{n}=(n+1 / 2) \hbar \omega_{\mathrm{c}}$. 


\section{Resonant tunnelling in narrow-well double-barrier structures}

In a DBS with narrow well (width $\leq 10 \mathrm{~nm}$ ) the effect of a transverse magnetic field is to broaden the resonant peak in $I(V)$ and shift it to higher voltages [6]. This is shown in Fig. 2 for an asymmetric $\mathrm{GaAs} / \mathrm{Al}_{0.4} \mathrm{Ga}_{0.6} \mathrm{As}$ structure with $6 \mathrm{~nm} \mathrm{QW}$, a thick emitter barrier $(11 \mathrm{~nm})$ and thin collector barrier $(8 \mathrm{~nm})$. Shifts

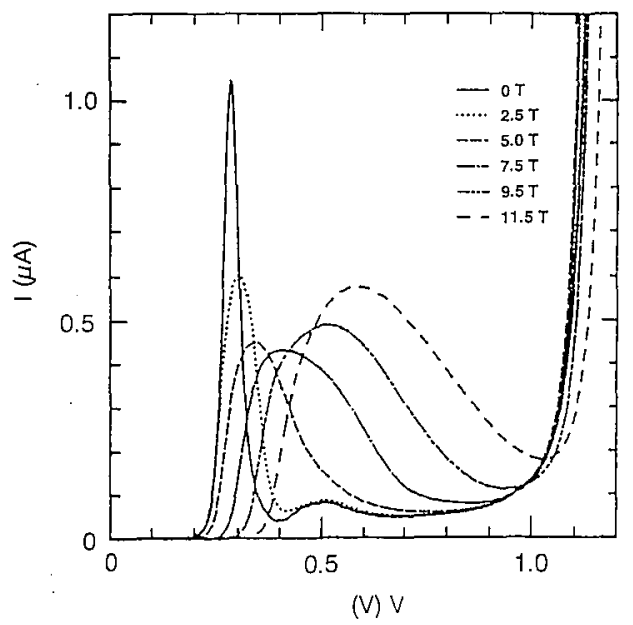

Fig. 2. Current-voltage characteristic $I(V)$ at $4 \mathrm{~K}$ for $\mathrm{GaAs} / \mathrm{Al}_{0.4} \mathrm{Ga}_{0.6}$ As double-barrier structure in transverse magnetic field $B=0$ to $B=11.5 \mathrm{~T}$. The structure is asymmetric with $6 \mathrm{~nm}$ well width, $11 \mathrm{~nm}$ emitter-barrier width and $8 \mathrm{~nm}$ collector-barrier width.

of the resonance due to electronic charge buildup in the QW can then be neglected and the resonant peak is very narrow [7]. In this case the magnetic potential is only a small perturbation on the subband states of the emitter 2DEG and QW. The energy of a state $\left(k_{y}, k_{z}\right)$ in the QW is then

$$
E_{\mathrm{w}}\left(k_{y}, k_{z}\right)=E_{\mathrm{w}}+\Delta E_{\mathrm{w}}+\frac{\hbar^{2}}{2 m^{*}}\left\{\left(k_{y}+k_{\mathrm{w}}\right)^{2}+k_{z}^{2}\right\},
$$

where $E_{\mathrm{w}}$ is the subband energy, $\Delta E_{\mathrm{w}}$ the diamagnetic shift ( $\leq 1 \mathrm{meV}$ for $B \approx$ $12 \mathrm{~T}$ in narrow-well DBS) and $\hbar k_{\mathrm{w}}=e B\langle x\rangle_{\mathrm{w}}$. The last term is the in-plane kinetic cnergy which is essentially $1 /\left(2 m^{*}\right) v_{y}^{2}$, with $m^{*} v_{y}=\hbar k_{y}+e B\langle x\rangle_{\mathrm{w}}$ when averaged over the subband wave function $\psi_{\mathrm{w}}(x)$. A similar result follows for the emitter 2DEG state with $\left(k_{y}, k_{z}\right)$ :

$$
E_{\mathrm{a}}\left(k_{y}, k_{z}\right)=E_{\mathrm{a}}+\Delta E_{\mathrm{a}}+\frac{\hbar^{2}}{2 m^{*}}\left\{\left(k_{y}+k_{\mathrm{a}}\right)^{2}+k_{z}^{2}\right\},
$$

where $\hbar k_{\mathrm{a}}=e B\langle x\rangle_{\mathrm{a}}$ is averaged over the emitter bound state $\psi_{\mathrm{a}}(x)$. Since the width of the accumulation layer in $\mathrm{GaAs}$ is $\approx 10 \mathrm{~nm}$, the diamagnetic shift is again small. Thus the effect of the magnetic field is to give a relative momentum shift of the dispersion curves (5) and (6) by $\hbar k_{0}=\hbar\left(k_{\mathrm{w}}-k_{\mathrm{a}}\right)=e B \Delta s$, where 
$\Delta s=\langle x\rangle_{\mathrm{w}}-\langle x\rangle_{\mathrm{a}}$ is the mean distance between 2D electrons in the accumulation layer and QW. Physically this is due to the action of the Lorentz force as the electron traverses the emitter tunnel barrier.

In Fig. 3 we have drawn the dispersion curves of the emitter and QW states (choosing $\langle x\rangle_{\mathrm{w}}=0$ for simplicity). The emitter dispersion curve has only been drawn for the occupied states $-k_{\mathrm{F}}<k_{y}-k_{0}<k_{\mathrm{F}}$, where $k_{\mathrm{F}}$ is the Fermi wave vector of the degenerate 2DEG. Since energy and transverse momentum are con-
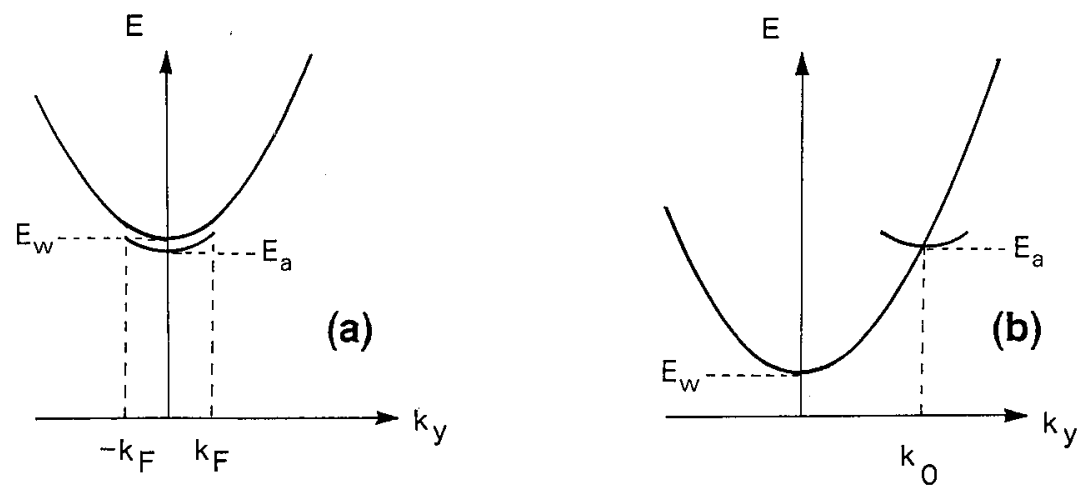

Fig. 3. In-plane dispersion curves $E$ versus $k_{y}$ for electrons in quantum well (subband minimum $E_{\mathrm{w}}$ ) and accumulation layer (subband minimum $E_{\mathrm{a}}$ ) for (a) zero magnetic field and (b) non-zero magnetic field, applied parallel to the layer interfaces.

served, tunnelling can only occur for those states at the intersection points of the two curves. For $B=0$ (Fig. 3a), this occurs for all occupied emitter states when $E_{\mathrm{a}}=E_{\mathrm{w}}$, giving rise to a sharp peak in $I(V)$. From Fig. 1 the voltage drop $V_{1}$ between emitter contact and well centre is then given by $e V_{1}=E_{\mathrm{w}}+E_{\mathrm{F}}$. For $B \neq 0$ (Fig. $3 \mathrm{~b}$ ) resonant tunnelling occurs over a range of biases which broadens the peak. The peak position corresponds roughly to the intersection at $k_{y}=k_{0}$, when $E_{\mathrm{a}}=E_{\mathrm{w}}+\hbar^{2} k_{0}^{2} /\left(2 m^{*}\right)$ and hence $e V_{1}=E_{\mathrm{w}}+E_{\mathrm{F}}+(e B \Delta s)^{2} /\left(2 m^{*}\right)$. Assuming the applied voltage $V \propto V_{1}$ over a small range, this predicts a voltage shift of the peak $\propto B^{2}$ in agreement with experiments [7-9].

Although detailed calculations of the $I(V)$ curves have not been carried out (owing to the uncertain effects of broadening processes), the qualitative picture is clear. Electrons in the accumulation layer with a well-defined range of transverse momenta, tunnel through the emitter barrier and are injected into the $Q W$ with a momentum distribution shifted by the magnetic field. Since their kinetic energy in the well is tuned by the applied voltage, the tunnelling electrons can be used as a spectroscopic probe of the energy and momentum spectrum of the states in the QW. We now discuss the application of this technique to investigate magnetoelectric states in wide wells, hole states in $p$-type structures and single impurity states in QW's. 


\section{Resonant tunnelling in wide-well double-barrier structures}

Experiments on structures with wide wells (width $w \approx 100 \mathrm{~nm}$ ) show series of closely-spaced, weak resonances [10]. This is expected since the level separation between QW subband decreases and the probability of scattering in the well increases width $w$. These structures are interesting because the character of the QW states can be substantially modified by a transverse magnetic field. At low fields they are traversing states in which an electron bounces back and forth between the confining barriers, but at high fields, with increasing orbit curvature, they become skipping states in which an electron bounces (skips) along a single interface (see insets in Fig. 6). Experimentally, the changing structure of the QW states is most clearly seen in the amplitudes of the magneto-oscillations in the second derivative plot of $\mathrm{d}^{2} I / \mathrm{d} B^{2}$ versus $B$ for fixed bias voltage. In Fig. 4 , three series of oscillations with different periodicities can be distinguished and the changeover from traversing to skipping states is revealed as a region of much reduced magneto-oscillation amplitude. It is significant that the tumnel current is reduced to zero at very high magnetic fields.

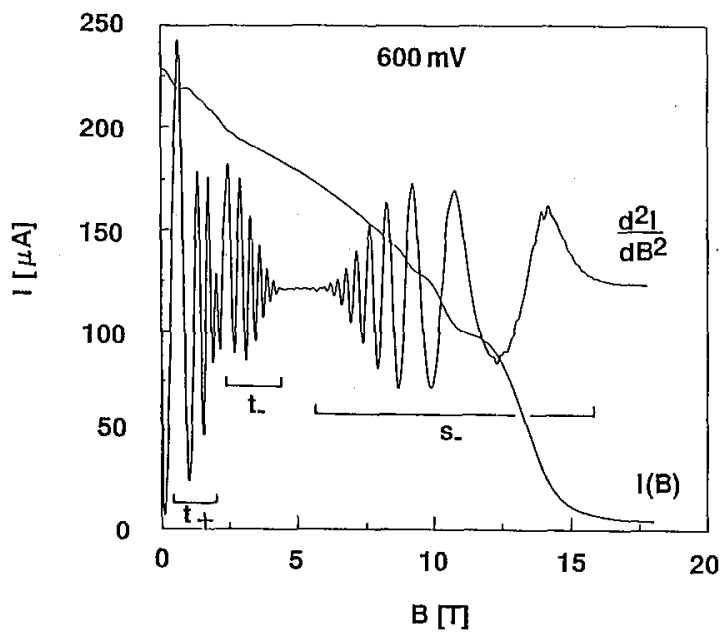

Fig. 4. Experimental results for magneto-oscillations in current $I$ and derivative $\mathrm{d}^{2} I / \mathrm{d} B^{2}$ versus field $B$ for wide-well $(120 \mathrm{~nm})$ double-barrier heterostructure measured at fixed bias voltage $600 \mathrm{mV}$ and at temperature $4 \mathrm{~K}$.

The spatial variation of electron potential energy $E_{\mathrm{c}}(x)$ is shown in Fig. 5 for the structure with $120 \mathrm{~nm}$ well described in Ref. [9]. We have modelled the accumulation layer using the Fang-Howard variational wave function. Owing to the electric field in the well, the injected electrons may attain kinetic energies of several hundred $\mathrm{meV}$ so it is important to take account of nonparabolicity in describing the electronic states. In a transverse magnetic field the classical orbits are cycloids, which may interact with either or both barrier interfaces. We have calculated the dispersion curves $E_{n}\left(k_{y}\right)$ for these states in the WKB approximation 


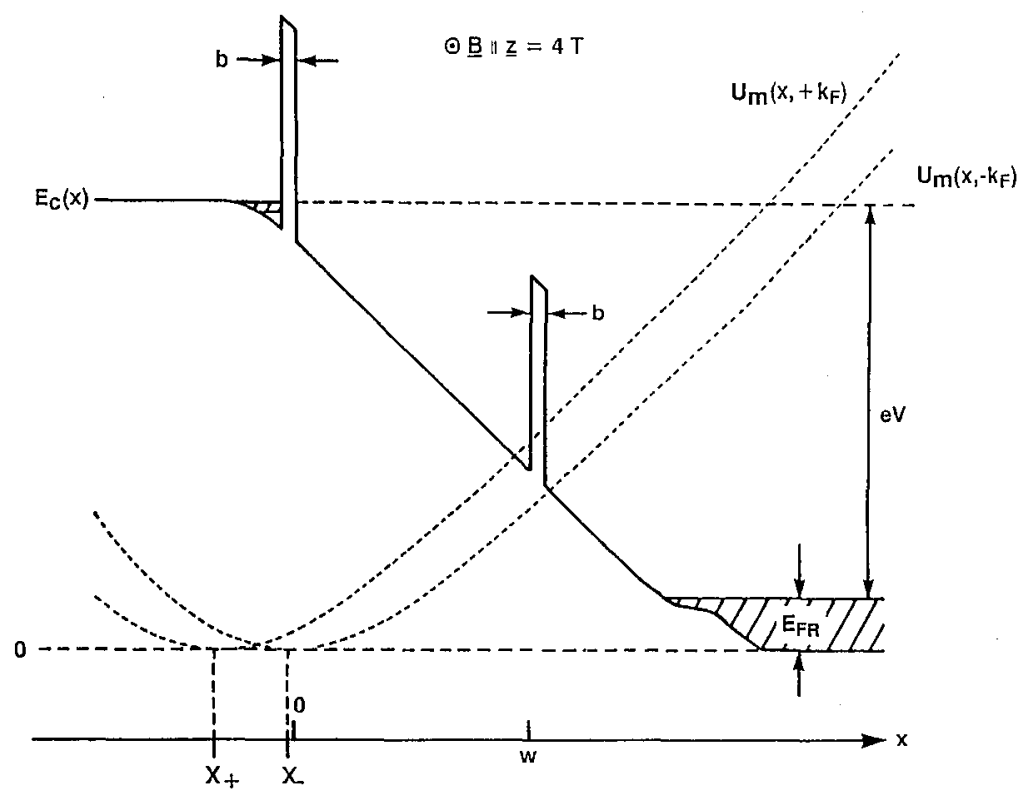

Fig. 5. Conduction-band profile for wide-well structure $(w=120 \mathrm{~nm}, b=5.6 \mathrm{~nm}$, $V=600 \mathrm{mV}$ ) and magnetic potential for $+k_{F} /-k_{F}$ electrons tunnelling into $n=13 / 21$ traversing state in well at $B=4 \mathrm{~T}$.

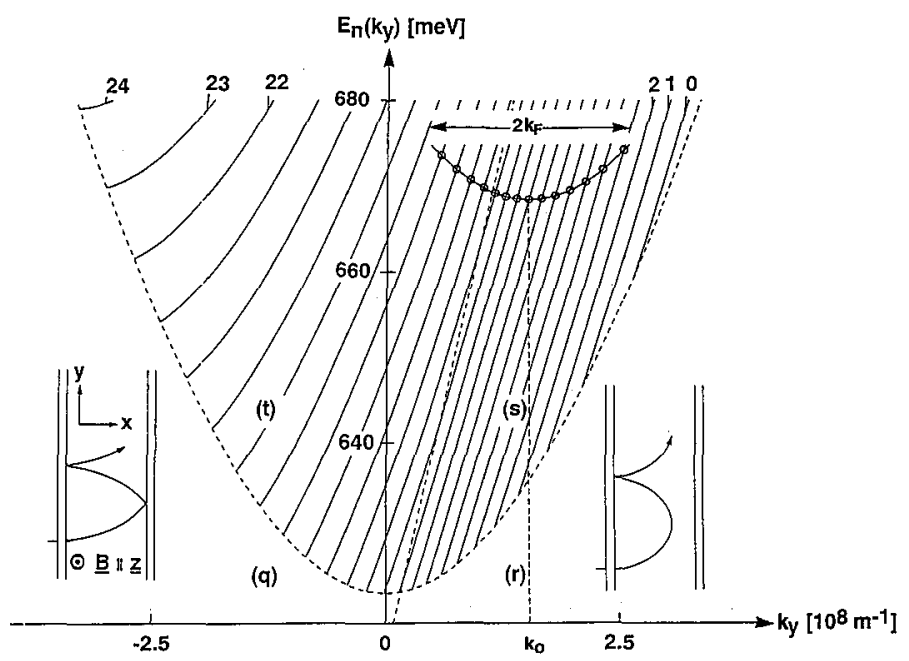

Fig. 6. Calculated dispersion curves $E_{n}\left(k_{y}\right)$ for cycloidal quantum-well states $(B=5 \mathrm{~T}$, $V=600 \mathrm{mV}$ ) including nonparabolicity and showing intersection points (open circles) with parabolic 2DEG dispersion curve. Lower insets show traversing orbits (left) and skipping orbits (right). 
[10]. In Fig. 6, region $s$ corresponds to emitter-bound skipping states, region $t$ to traversing states. Regions $q$ and $r$ (collector-bound and bulk states) are inaccessible to electron tunnelling from the emitter 2DEG. The parabolic dispersion curve of the occupied 2DEG states is centred on $k_{y}=k_{0}=e B(b+3 a) / \hbar$, where $b$ is the barrier width and $3 a$ the offset distance of the 2DEG from the barrier interface. Here, the origin of coordinates is at the right hand side of the emitter barrier so that $\hbar k_{y}$ has the physical interpretation of the transverse momentum of an electron as it emerges from the tunnel barrier (from Eq. (2), $p_{y}=m^{*} v_{y}$ when $x=0)$. Energy and momentum conservation then defines the allowed tunnelling channels by the intersection points $k_{y}=k_{y}(n)$ for the 2DEG and QW dispersion curves. With increasing $B$ the opening of a channel when $k_{y}(n)=k_{0}+k_{\mathrm{F}}$ and closure when $k_{y}(n)=k_{0}-k_{\mathrm{F}}$ gives rise to changes of slope in the $I(B)$ dependence which produce the pronounced magneto-oscillations in the $\mathrm{d}^{2} I / \mathrm{d} B^{2}$ versus $B$ plot.

However, to account for the distinctive features of the oscillation amplitudes it is necessary to calculate the field dependence of the tunnel current $I$ [11]. We use the sequential tunnelling approach described previously and write

$$
I=\frac{e}{2 \pi^{2}} \sum_{n} \iint \mathrm{d} k_{y} \mathrm{~d} k_{z}\left(f_{k}^{\mathrm{a}}-f_{n, k}^{\mathrm{w}}\right) \frac{2 \pi}{\hbar}\left|M_{n} k_{y}\right|^{2} \delta(\Delta E),
$$

where $M_{n}\left(k_{y}\right)$ is the Bardeen transfer matrix element [11] and $f^{\mathrm{a}}, f^{\mathrm{w}}$ are the occupancies of the 2DEG and QW states. For $f^{\mathrm{a}}$ we use a zero-temperature Fermi-Dirac distribution function since the degenerate 2DEG is maintained close to equilibrium by rapid diffusion through the emitter contact. In wide-well structures charge buildup in the $\mathrm{QW}$ is expected to be small $\left(f^{\mathrm{w}}=0\right)$ since the collector barrier is much more transparent than the emitter barrier. We note also that when the electrons tunnel into skipping states confined against the emitter barrier, scattering processes are essential to maintain current continuity through the structure. In Eq. (7) the integration over $k_{y}$ is accomplished using the conservation of energy condition $\delta(\Delta E)=0$, where $\Delta E$ is the energy difference between an emitter $2 \mathrm{D}$ state and cycloidal QW state with the same $k_{y}$ and $k_{z}$. The tunnel current is then the sum of contributions $I_{n}$ due to each conduction channel,

$$
I_{n}=\frac{2 e}{\pi \hbar}\left[\frac{\left\{k_{\mathrm{F}}^{2}-\left(k_{y}-k_{0}\right)^{2}\right\}^{1 / 2}\left|M_{n}\left(k_{y}\right)\right|^{2}}{\left|\hbar^{2}\left(k_{y}-k_{0}\right) / m^{*}-\mathrm{d} E_{n} / \mathrm{d} k_{y}\right|}\right]_{k_{y}=k_{y}(n)} .
$$

The Bardeen transfer matrix element is given by [12]

$$
M_{n}\left(k_{y}\right)=\frac{\hbar^{2}}{2 m^{*}}\left(\psi_{\mathrm{a}} \frac{\mathrm{d} \psi_{n}^{*}}{\mathrm{~d} x}-\psi_{n}^{*} \frac{\mathrm{d} \psi_{\mathrm{a}}}{\mathrm{d} x}\right)_{x=0} .
$$

Using WKB states for the emitter bound state $\psi_{\mathrm{a}}$ and QW states $\psi_{n}$, we have shown that $[11,13]$

$$
\left|M_{n}\left(k_{y}\right)\right|^{2}=\hbar^{2} F_{\mathrm{a}} F_{n}\left(k_{y}\right) T_{n}\left(k_{y}\right),
$$

where $F_{\mathrm{a}}$ is the attempt rate (number of collisions per second with the emitter barrier) of a 2DEG electron and $T_{n}\left(k_{y}\right)$ is the emitter barrier transmission coefficient, which depends on $k_{y}$ via the influence of the magnetic potential on the tunnel barrier. For an electron in the $n$-th $\mathrm{QW}$ state the attempt rate $F_{n}\left(k_{y}\right)$ is given by

$$
\frac{1}{F_{n}\left(k_{y}\right)}=\int \frac{\mathrm{d} x}{\left|v_{n}\left(k_{y}, x\right)\right|},
$$


where $v_{n}\left(k_{y}, x\right)$ is the local velocity and the integral is over the classical orbit between successive collisions with the emitter barrier.

The calculated current contributions $I_{n}(B)$ are shown in Fig. 7 for a $120 \mathrm{~nm}$ well structure with $5.6 \mathrm{~nm}$ barriers. To simulate broadening effects we have averaged the total current over a small range of $B$. The $\mathrm{d}^{2} I / \mathrm{d} B^{2}$ plot thus obtained shows three distinct series of oscillations as found experimentally. The low field $t+/ t-$ series originate from the opening/closure of traversing state channels. The high field $s-$ series is due to tunnelling of $-k_{F}$ electrons into emitter-bound cycloidal skipping states. The periodicities of these series are quite well described by

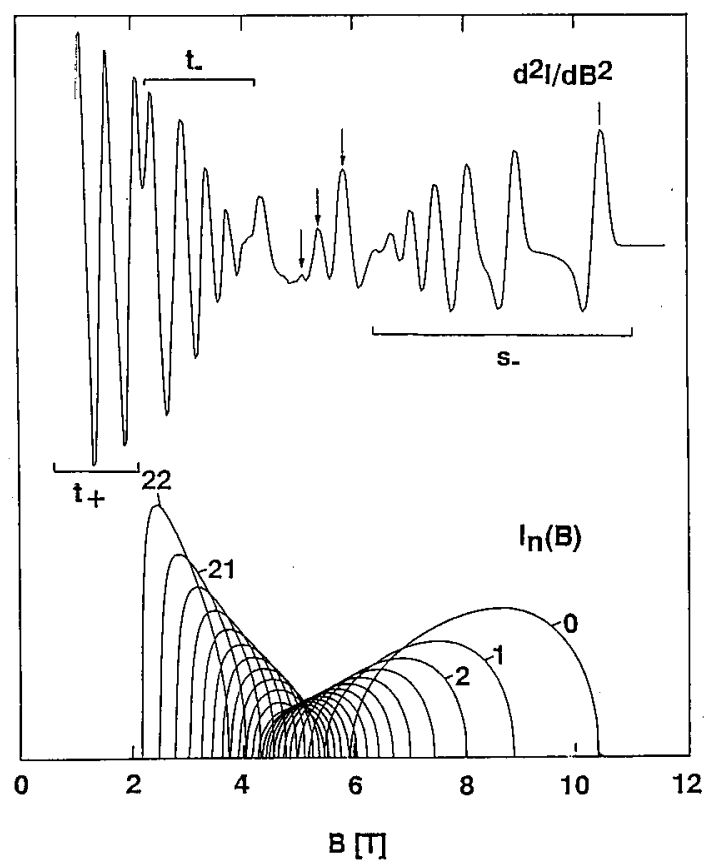

Fig. 7. Calculated magneto-oscillations in $\mathrm{d}^{2} I / \mathrm{d} B^{2}$ versus $B$ for structure of Fig. 5 showing $t-, t+, s-$ and weak $s+$ (arrows) series. Lower curves give current contributions $I_{n}(B)$ for tunnelling into $n=0,1,2, \ldots, 22$ cycloidal well states.

our nonparabolic WKB approximation but the presence of weak $s+$ oscillations, not seen experimentally, indicates the limitations of the model in determining the energy spectrum for low quantum numbers $n$.

The changeover from traversing to skipping state resonances is characterised by a reduction in the current contributions $I_{n}$ and hence in reduced oscillation amplitudes in $\mathrm{d}^{2} I / \mathrm{d} B^{2}$. This behaviour has a direct physical interpretation in terms of the field dependence of the attempt rate $F_{n}\left(k_{y}\right)$ of an electron in a QW state as shown in Fig. 8. For traversing states, as $B$ increases, the increasing orbit curvature increases the path length and time interval between collisions with the well walls. This decreases $F_{n}$ and also the oscillatory amplitudes. By contrast, for 


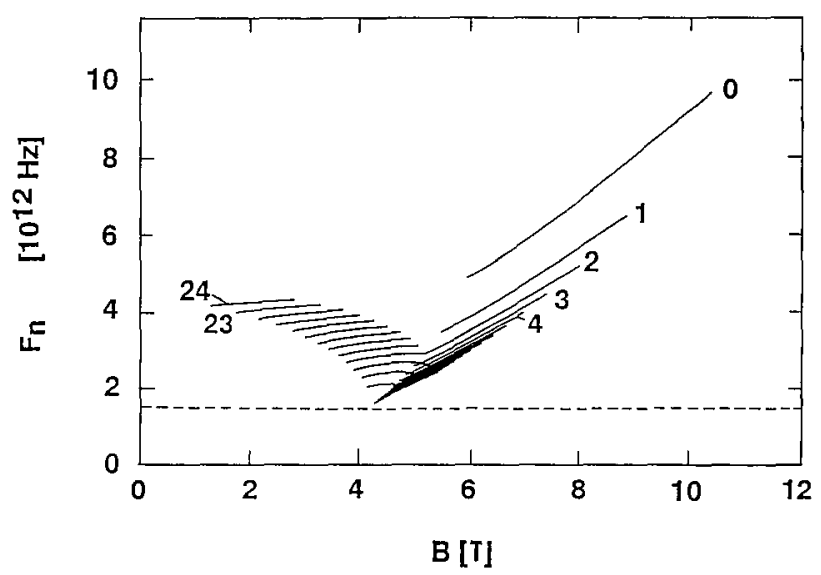

Fig. 8. Field dependence of attempt rate $F_{n}$ for tunnelling into $n=0,1,2, \ldots, 24$ cycloidal well states. Broken line gives approximate LO phonon cmission rate in bulk GaAs.

skipping states, as $B$ increases the electron orbit is pulled closer to the emitter barrier interface. This shortens the distance between successive skips which increases the attempt rate and hence oscillatory amplitudes. At very high $B$ the electrons are deflected back into the emitter before they can escape into the well so the current is reduced to zero. The longest trajectories in the well and hence lowest attempt rate $F_{\min } \approx 2 \times 10^{12} \mathrm{~s}^{-1}$, occur in the changeover region when the electron impinges at grazing incidence on the collector barrier interface. The current contributions are then small and also level broadening is more important since $F_{\min }$ is comparable to the LO phonon emission rate in GaAs. Thus the magneto-oscillations are weak and poorly resolved as observed.

For the thin barriers $(5.6 \mathrm{~nm})$ used in this study the effect of the magnetic field on the transmission coefficient $T_{n}\left(k_{y}\right)$ is small. For wider barriers the field dependence of the tunnelling probability can have dramatic effects as found in a previous study of skipping states in single-barrier structures $[13,14]$.

\section{Resonant tunnelling of holes in p-type narrow-well double-barrier structures}

Resonant tunnelling of holes has been observed in GaAs/AlAs double-barrier structures with $p$-type contact layers. Figure 9a shows recent experimental results [15] for a structure with $4.2 \mathrm{~nm} \mathrm{QW}$ grown on (100) planes, in which six resonances are clearly visible. The variation of hole potential energy through the structure is given schematically in Fig. 9b. More resonances are observed than in similar $n$-type structures because there are QW subbands associated with heavy holes (III's) and light holes (LH's), both of which have rather larger effective masses than conduction electrons in GaAs. Also the voltage shifts of the hole resonant peaks in a transverse magnetic field behave quite differently to their electronic 
counterparts. As can be seen in Fig. 9a, some peaks shift very little while others shift in opposite directions.
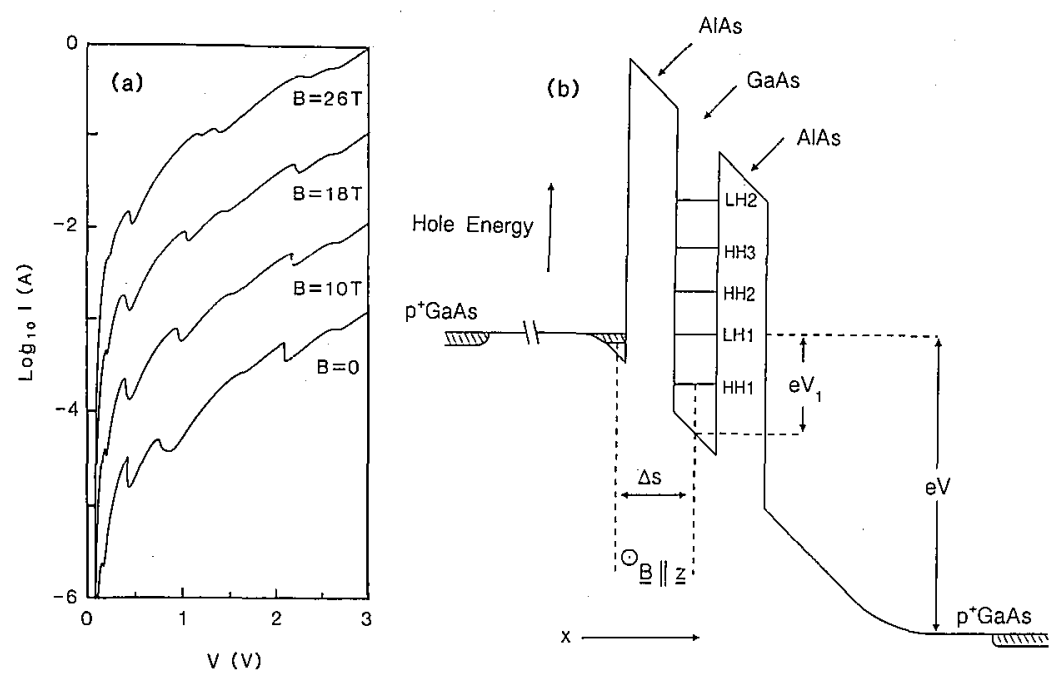

Fig. 9. (a) Logarithmic plot of current-voltage $I(V)$ characteristic of $p$-type double-barrier structure (well width $4.2 \mathrm{~nm}$ ) for various transverse magnetic fields measured at $4 \mathrm{~K}$; (b) Schematic diagram of valence-band profile when under bias voltage $V$ showing formation of two-dimensional hole gas adjacent to emitter barrier. Note that hole energy is plotted upwards.

In the electronic case the quadratic voltage shift with field $B$ is due to the parabolic dispersion of the QW subband states in the effective-mass approximation. The in-plane dispersion curves of hole subbands are more complicated due to the mixing of HH and LH states [16]. These are decoupled in the bulk semiconductor but coupled in a heterostructure due to the confining QW potential, except at $k_{y}=k_{z}=0$. In Fig. $9 \mathrm{~b}$ the subbands are labelled according to their character at $k_{y}=k_{z}=0$. Despite these complications the basic principles elucidated in Sec. 2 still apply (with change of $-e$ to $e$ ). In lowest-order perturbation theory the effect of the magnetic field is to replace $\hat{p}_{y}$ by $\hbar k_{y}-e B\langle x\rangle_{\mathrm{w}}$. If the $\mathrm{QW}$ is symmetric the mean hole position is in the middle of the $\mathrm{QW}$ as in the electronic case. Owing to the more complicated hole IIamiltonian [17], the diamagnetic shifts are wave vector dependent but are still small $(\approx 0.5 \mathrm{meV})$ even at the large fields used experimentally $(B \approx 26 \mathrm{~T})$.

Thus the voltage and field positions of the resonant peak positions, plotted in Fig. 10a, give a map of the dispersion curves of the hole subbands and reveals the large nonparabolicity and regions with negative in-plane effective mass. As shown in Fig. 9b tunnelling takes place from the HH states of a 2DIIG in the emitter. The $B$ scale of Fig. 10a may therefore be converted to a momentum scale using $k_{y}=k_{0}=e B \Delta s / \hbar, \Delta s=\lambda+b+1 / 2 w$, where $\lambda$ is the offset distance of the emitter 2DHG from the barrier of width $b$. Using $\lambda=5 \mathrm{~nm}$ and $b=5.1 \mathrm{~nm}$ 
the upper scale of Fig. 10a is obtained. For the same range of $k_{y}$ the in-plane dispersion curves have been calculated [15] from the Luttinger Hamiltonian [17] and are shown in Fig. 10b. It can be seen that the theoretical curves reproduce qualitatively all the main features of the experimental results. To scale the applied voltage $V$ to the subband energies requires a band bending model in which there are uncertainties due to dopant diffusion and the eflect of charge buildup in the $\mathrm{QW}$ at resonance. Estimates [15] give scale factors $\approx 3$ at $1 \mathrm{~V}$ and $\approx 5$ at $3 \mathrm{~V}$ in broad agreement with experimental results.
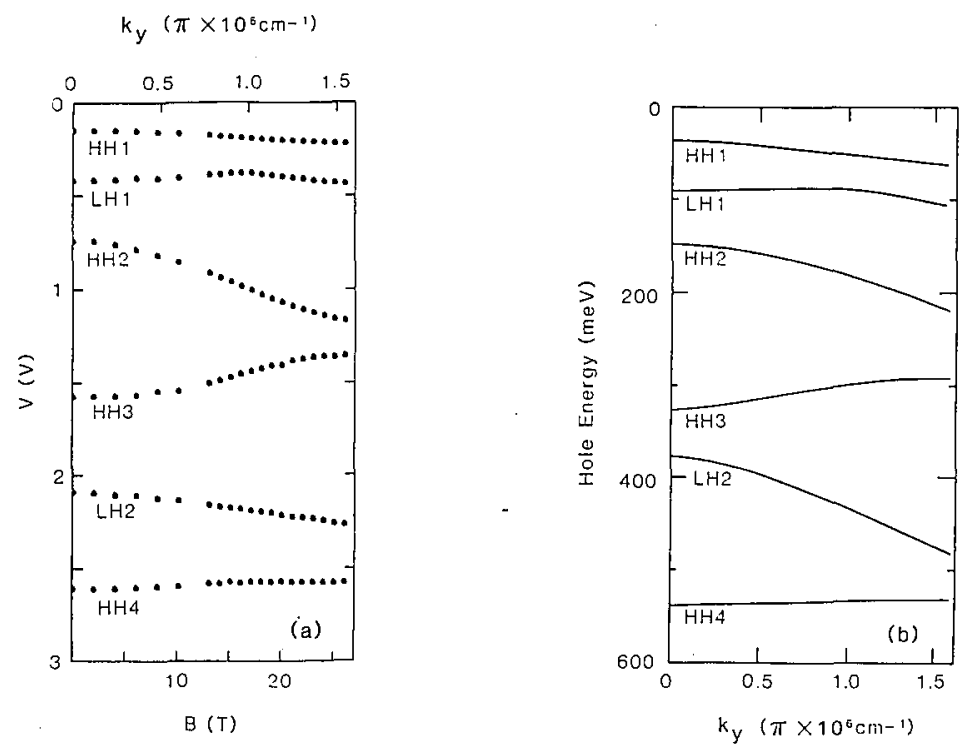

Fig. 10. Dependence of voltage positions of resonant peaks in $I(V)$ on transverse magnetic field $B$; (b) Calculated in-plane dispersion curve for isolated AlAs/GaAs/AlAs quantum well of width $4.2 \mathrm{~nm}$ in zero electric and magnetic fields.

Further work [18], in which the charge buildup in the QE was directly measured (from magneto-oscillation with $\boldsymbol{B}$ perpendicular to the layers), have provided a more accurate scaling of the applied voltage to the hole energy. By rotating the magnetic field in the (100) plane of the QW, small cubic anisotropy effects were also found in the hole dispersion curves. More dramatic anisotropy effects have been found in resonant magneto-tunnelling studies of the hole subbands of strained $\mathrm{Si} / \mathrm{Si}_{1-x} \mathrm{Ge}_{x}$ QWs [19] and in GaAs/AlAs QWs grown on (311)A substrates [20].

\section{Resonant tunnelling into isolated donor states in a quantum well}

Recent experiments on a DBS with small cross-sectional area $\left(\approx 0.5 \mu \mathrm{m}^{2}\right)$ have shown very weak peaks in the $I(V)$ curves at voltages well below the main resonant peak for tunnelling into the lowest QW subband (Fig. 11). It has been proposed that these are due to resonant tunnelling into the localized bound states 


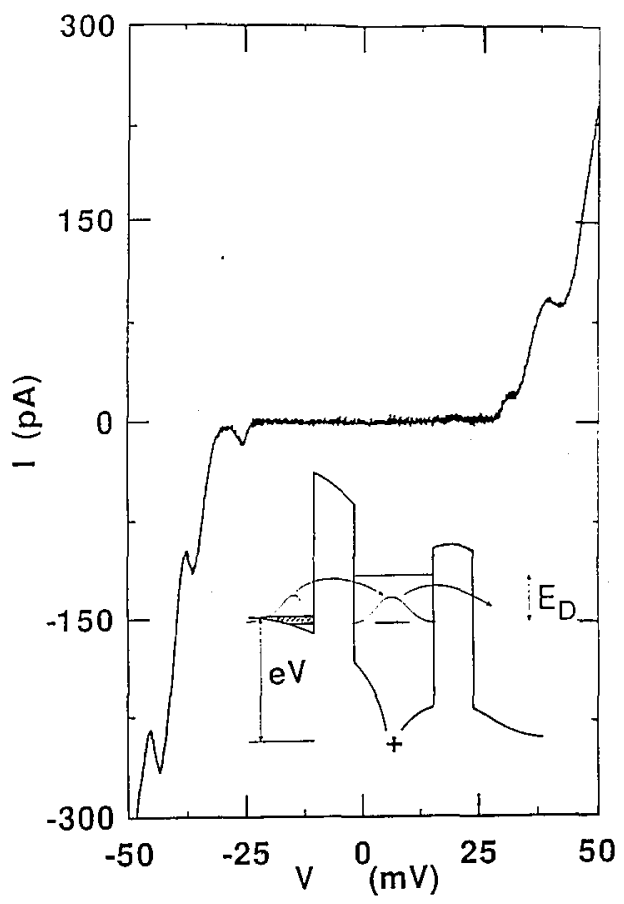

Fig. 11. Current-voltage characteristic $I(V)$ of small-area $\mathrm{GaAs} / \mathrm{Al}_{0.1} \mathrm{Ga}_{0.6} \mathrm{As}$ double-barrier structure (well width $12 \mathrm{~nm}$, barrier widths $5.7 \mathrm{~nm}$ ) for voltages below main resonant tunnelling peak, measured at temperature $39 \mathrm{mK}$. Lower inset shows schematic band profile for resonant tunnelling from 2DEG via localized donor state in well.

of a small number of donor impurities in the nominally undoped QW [21]. We now consider what information the magnetotunnelling technique will provide in this situation [22].

For tunnelling into a localized donor state, the transverse components of momentum $k_{y}, k_{z}$ are not conserved. Energy conservation then shows that tunnelling only occurs for the small range of bias voltages for which the donor level lies within the energy range of occupied 2D states in the emitter accumulation layer. At a particular bias the kinetic energy of the resonant 2D states is (from Fig. 11 and Fig. 1) $E_{\mathrm{c}}=E_{\mathrm{w}}-E_{\mathrm{D}}-E_{\mathrm{a}}$, where $E_{\mathrm{D}}$ is the donor binding energy below the subband minimum at $E_{\mathrm{w}}$. Taking a separable form $\phi(y, z) \chi(x)$ for the donor wave function [23], the Bardeen matrix element will contain an overlap integral (evaluated in the tunnel barrier),

$$
S\left(k_{y}, k_{y}\right)=\iint \phi(y, z) \exp \mathrm{i}\left(k_{y} y+k_{z} z\right) \mathrm{d} y \mathrm{~d} z
$$

between the emitter plane-wave state $\left(k_{y}, k_{z}\right)$ and the lateral donor wave function. The tunnelling rate is proportional to the momentum probability distribution function $P\left(k_{y}, k_{z}\right)=\left|S\left(k_{y}, k_{z}\right)\right|^{2}$. 
In a transverse magnetic field, owing to the action of the Lorentz force, the required momentum distribution becomes $P\left(k_{y}+k_{0}, k_{z}\right)$, which is shifted by $k_{0}=$ $e B \Delta s / \hbar$, where $\Delta s$ is mean distance between emitter 2DEG and donor site. The field dependence of the tunnel current $I(B)$ is obtained by averaging over values of $k_{y}, k_{z}$ which satisfy the energy conservation condition $k_{y}^{2}+k_{z}^{2}=k_{\mathrm{c}}^{2}=2 m^{*} E_{\mathrm{c}} / \hbar^{2}$. This gives

$$
I(B) \propto \int_{-k_{\mathrm{c}}}^{k_{\mathrm{c}}} \frac{P\left(k_{y}+k_{0}, k_{z}\right) \mathrm{d} k_{y}}{\left(k_{\mathrm{c}}^{2}-k_{y}^{2}\right)^{1 / 2}} .
$$

The integral may be evaluated numerically for various model wave functions. However, if the lateral width $\Delta x$ of the donor wave function obeys $k_{\mathrm{c}} \Delta x \ll 1$, it is clear that $I(B) \propto P\left(k_{0}, 0\right)$. In this case the field dependence $I(B)$ gives directly the spatial Fourier transform and hence spatial width of the localized donor state, provided that the donor wave function is not appreciably perturbed by the magnetic field. For a Gaussian model wave function $\phi(y, z) \approx \exp \left[-\left(y^{2}+z^{2}\right) / 2 a_{0}^{2}\right]$, with width parameter $a_{0}, I(B) \approx \exp \left(-k_{0}^{2} a_{0}^{2}\right)$, so the impurity peak is suppressed for a sufficiently large field. This effect has been observed in more recent experiments on resonant tunnelling structures in which the QW is weakly delta doped in the centre [24].

\section{Conclusion}

We have described how magneto-tunnelling in a transverse field is a remarkable tool for the study of excitations in semiconductor heterostructures. In contrast to other techniques, such as optical measurements, it directly probes states with specific in-plane $k$ values and, by rotating the magnetic field, the anisotropy between different crystal directions is revealed. By using very high magnetic fields $(40 \mathrm{~T}) k$ values $\approx 10^{7} \mathrm{~cm}^{-1}$ have been reached, so a substantial part of the Brillouin zone is a vailable for study.

Unfortunately, this technique is not available in other areas of solid-state physics in which tunnelling is important such as the scanning tunnelling microscope and normal metal or superconducting tunnel junctions. It is only in semiconductor heterostructures prepared by modern growth techniques that such perfect surfaces are achieved that transverse momentum is conserved, and where sufficiently wide and low tunnel barriers are available to give a large momentum shift without reducing the tunnel current to a negligible level. We should be grateful for these features and seek to exploit them further.

\section{Acknowledgments}

This work is supported by the UK Science and Engineering Research Council. We are grateful to Dr. P.H. Beton and Prof. L. Eaves for stimulating discussions. 


\section{References}

[1] B. Ricco, M.Ya. Azbel, Phys. Rev. B 29, 1970 (1984).

[2] F. Capasso, S. Sen, F. Beltram, A.Y. Cho, in: Physics of Quantum Electron Devices, Ed. F. Capasso, Springer Series in Electronics and Photonics, Vol. 28, Chap. 7, Springer Verlag, Berlin, Heidelberg 1990.

[3] F.W. Sheard, G.A. Toombs, Semicond. Sci. Technol. 7, B460 (1992).

[4] S. Luryi, Appl. Phys. Lett. 47, 490 (1985).

[5] F.W. Sheard, K.S. Chan, G.A. Toombs, L. Eaves, in: 14 th Intern. Symposium on GaAs and Related Compounds (Crete 1987), Institute of Physics Conf. Series 91, 387 (1988).

[6] L. Eaves, R.K. Hayden, M.L. Leadbeater, D.K. Maude, E.C. Valadares, M. IIenini, F.W. Sheard, O.H. Hughes, J.C. Portal, L. Cury, Surf. Sci. 263, 199 (1992).

[7] M.L. Leadbeater, E.S. Alves, F.W. Sheard, L. Eaves, M. Henini, O.H. Hughes, G.A. Toombs, J. Phys., Condens. Matter 1, 10605 (1989).

[8] M.L. Leadbeater, L. Eaves, P.E. Simmonds, G.A. Toombs, F.W. Sheard, P.A. Claxton, G. Hill, M.A. Pate, Solid-State Electron. 31, 707 (1988).

[9] S. Ben Amor, K.P. Martin, J.J.L. Rascol, R.J. Higgins, A. Torabi, H.M. IIarris, J.C. Summers, App. Phys. Lett. 53, 2540 (1988).

[10] M.L. Leadbeater, E.S. Alves, L. Eaves, M. Henini, O.H. Iughes, A. Celeste, J.C. Portal, G. Hill, M.A. Pate, J. Phys. Condens. Malter 1, 4865 (1989); See also L. Eaves, E.S. Alves, T.J. Foster, M. Henini, O.H. Hughes, M.L. Leadbeater, F.W. Sheard, G.A. Toombs, K.S. Chan, A. Celeste, J.C. Portal, G. Hill, M.A. Pate, in: Physics and Technology of Submicron Structures, Eds. H. Heinrich, G. Bauer, F. Kuchar, Springer Series in Solid-State Sciences, Vol. 83, Springer Verlag, Berlin 1988.

[11] T.M. Fromhold, F.W. Sheard, G.A. Toombs, Proc. 20th Intern. Conf. on Physics of Semiconductors (Thessaloniki, Greece 1990), Eds. E.M. Anastassakis, J.D. Joannopoulos, World Scientific, Singapore 1990, p. 1250.

[12] J. Bardeen, Phys. Rev. Lett. 6, 57 (1961).

[13] T.M. Fromhold, Ph. D. Thesis, University of Nottingham, Nottingham 1990.

[14] T.M. Fromhold, F.W. Sheard, G.A. Toombs, Surf. Sci. 228, 437 (1990).

[15] R.K. Hayden, D.K. Maude, L. Eaves, E.C. Valadares, M. Henini, F.W. Sheard, O.H. Hughes, J.C. Portal, L. Cury, Phys. Rev. Lett. 66, 1749 (1991).

[16] L.C. Andreani, A. Pasquarello, F. Bassani, Phys. Rev. B 36, 5887 (1987).

[17] J.M. Luttinger, Phys. Rev. 102, 1030 (1956).

[18] R.K. Hayden, T. Takamasu, D.K. Maude, E.C. Valadares, L. Eaves, U. Ekenberg, N. Miura, M. Henini, J.C. Portal, G. Hill, M.A. Pate, Semicond. Sci. Technol. 7, B413 (1992).

[19] U. Gennser, V.P. Kesan, D.A. Syphers, T.P. Smith, S.S. Iyer, E.S. Yang, Phys. Rev. Lett. 67, 3828 (1991).

[20] R.K. Hayden, E.C. Valadares, M. Henini, L. Eaves, D.K. Maude, J.C. Portal, Phys. Rev. $B$, to be published.

[21] M.W. Dellow, P.H. Beton, C.J.G.M. Langerak, T.J. Foster, P.C. Main, L. Eaves, M. Henini, S.P. Beaumont, C.D.W. Wilkinson, Phys. Rev. Lett. 68, 1754 (1992).

[22] T.M. Fromhold, F.W. Sheard, L. Eaves, Acta. Phys. Pol. A 82, (1992). 
[23] R.L. Greene, K.K. Bajaj, Solid State Commun. 45, 825 (1983).

[24] J.W. Sakai, T.M. Fromhold, P.H. Beton, M. Henini, L. Eaves, P.C. Main, F.W. Sheard, G. Hill, submitted to 3rd Yamada Intern. Conf. on Application of High Magnetic Fields (Chiba, Japan 1992). 